\title{
Enhanced energy density with a wide thermal stability in epitaxial $\mathrm{Pb}_{0.92} \mathrm{La}_{0.08} \mathrm{Zr}_{0.52} \mathrm{Ti}_{0.48} \mathrm{O}_{3}$ thin films
}

Guangliang Hu, Chunrui Ma, Wei Wei, Zixiong Sun, Lu Lu, Shao-Bo Mi, Ming Liu, Beihai Ma, Judy Wu, and Chun-lin Jia

Citation: Appl. Phys. Lett. 109, 193904 (2016);

View online: https://doi.org/10.1063/1.4967223

View Table of Contents: http://aip.scitation.org/toc/apl/109/19

Published by the American Institute of Physics

\section{Articles you may be interested in}

Research Update: Enhanced energy storage density and energy efficiency of epitaxial

$\mathrm{Pb}_{0.9} \mathrm{La}_{0.1}\left(\mathrm{Zr}_{0.52} \mathrm{Ti}_{0.48}\right) \mathrm{O}_{3}$ relaxor-ferroelectric thin-films deposited on silicon by pulsed laser deposition APL Materials 4, 080701 (2016); 10.1063/1.4961636

Temperature-dependent energy storage properties of antiferroelectric $\mathrm{Pb}_{0.96} \mathrm{La}_{0.04} \mathrm{Zr}_{0.98} \mathrm{Ti}_{0.02} \mathrm{O}_{3}$ thin films Applied Physics Letters 104, 263902 (2014); 10.1063/1.4887066

Thickness-dependent dielectric and energy storage properties of $\left(\mathrm{Pb}_{0.96} \mathrm{La}_{0.04}\right)\left(\mathrm{Zr}_{0.98} \mathrm{Ti}_{0.02}\right) \mathrm{O}_{3}$ antiferroelectric thin films

Journal of Applied Physics 119, 124106 (2016); 10.1063/1.4944802

Enhanced energy storage density by inducing defect dipoles in lead free relaxor ferroelectric $\mathrm{BaTiO}_{3}$-based ceramics

Applied Physics Letters 110, 132902 (2017); 10.1063/1.4979467

$\mathrm{BiFeO}_{3}$-doped $\left(\mathrm{K}_{0.5}, \mathrm{Na}_{0.5}\right)\left(\mathrm{Mn}_{0.005}, \mathrm{Nb}_{0.995}\right)_{3}$ ferroelectric thin film capacitors for high energy density storage applications

Applied Physics Letters 110, 152901 (2017); 10.1063/1.4980113

High recoverable energy density over a wide temperature range in $\mathrm{Sr}$ modified $(\mathrm{Pb}, \mathrm{La})(\mathrm{Zr}, \mathrm{Sn}, \mathrm{Ti}) \mathrm{O}_{3}$

antiferroelectric ceramics with an orthorhombic phase

Applied Physics Letters 109, 262901 (2016); 10.1063/1.4973425

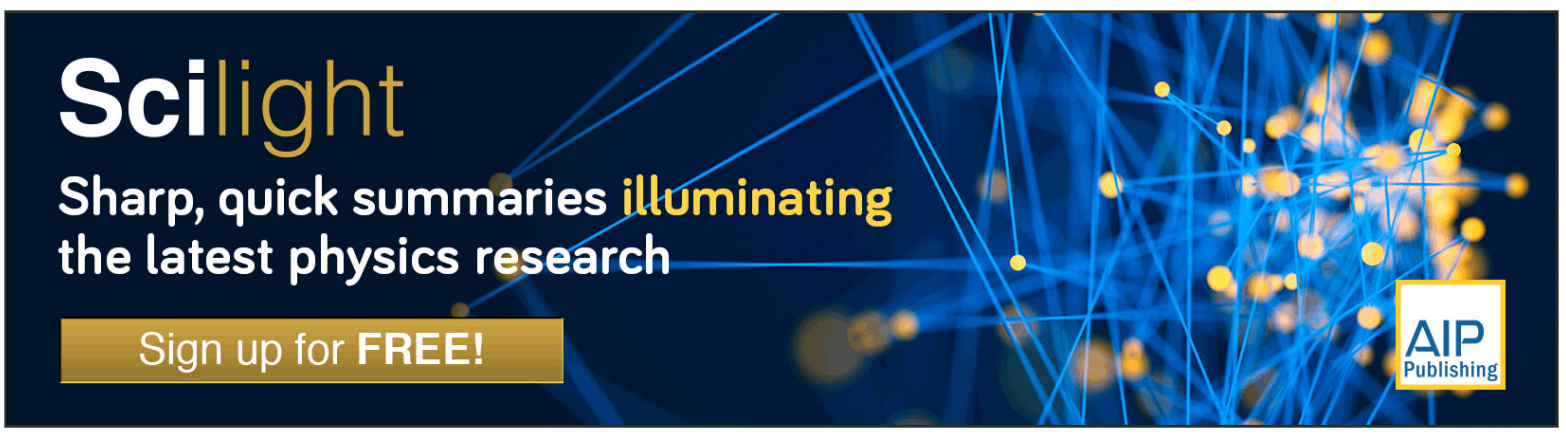




\title{
Enhanced energy density with a wide thermal stability in epitaxial $\mathrm{Pb}_{0.92} \mathrm{La}_{0.08} \mathrm{Zr}_{0.52} \mathrm{Ti}_{0.48} \mathrm{O}_{3}$ thin films
}

\author{
Guangliang Hu, ${ }^{1,2,3}$ Chunrui Ma, ${ }^{1, a)}$ Wei Wei, ${ }^{2}$ Zixiong Sun, ${ }^{2}$ Lu Lu, ${ }^{2}$ Shao-Bo Mi, ${ }^{1}$ \\ Ming Liu, ${ }^{2, a)}$ Beihai Ma, ${ }^{4}$ Judy Wu, ${ }^{3}$ and Chun-lin $\mathrm{Jia}^{2}$ \\ ${ }^{1}$ State Key Laboratory for Mechanical Behavior of Materials, Xi' an Jiaotong University, Xi' an 710049 , \\ People's Republic of China \\ ${ }^{2}$ School of Electronic and Information Engineering, Xi' an Jiaotong University, Xi' an 710049, China \\ ${ }^{3}$ Department of Physics and Astronomy, University of Kansas, Lawrence, Kansas 66045, USA \\ ${ }^{4}$ Energy Systems Division, Argonne National Laboratory, Argonne, Illinois 60439, USA
}

(Received 10 August 2016; accepted 25 October 2016; published online 8 November 2016)

\begin{abstract}
High-quality epitaxial $\mathrm{Pb}_{0.92} \mathrm{La}_{0.08} \mathrm{Zr}_{0.52} \mathrm{Ti}_{0.48} \mathrm{O}_{3}$ (PLZT) films of thickness of $\sim 880 \mathrm{~nm}$ were fabricated using pulsed laser deposition on (001) Nb doped $\mathrm{SrTiO}_{3}(\mathrm{Nb}: \mathrm{STO})$ substrates. Besides a confirmation of the epitaxial relationship $[100]_{\mathrm{PLZT}} / /[100]_{\mathrm{Nb}: \mathrm{STO}}$ and $(001)_{\mathrm{PLZT}} / /(001)_{\mathrm{Nb}: \mathrm{STO}}$ using $\mathrm{X}$-ray diffraction, a transmission electron microscopy study has revealed a columnar structure across the film thickness. The recoverable energy density $\left(W_{\text {rec }}\right)$ of the epitaxial PLZT thin film capacitors increases linearly with the applied electric field and the best value of $\sim 31 \mathrm{~J} / \mathrm{cm}^{3}$ observed at $2.27 \mathrm{MV} / \mathrm{cm}$ is considerably higher by $41 \%$ than that of the polycrystalline PLZT film of a comparable thickness. In addition to the high $W_{\text {rec }}$ value, an excellent thermal stability as illustrated in a negligible temperature dependence of the $W_{\text {rec }}$ in the temperature range from room temperature to $180^{\circ} \mathrm{C}$ is achieved. The enhanced $W_{\text {rec }}$ and the thermal stability are attributed to the reduced defects and grain boundaries in epitaxial PLZT thin films, making them promising for energy storage applications that require both high energy density, power density, and wide operation temperatures. Published by AIP Publishing. [http://dx.doi.org/10.1063/1.4967223]
\end{abstract}

Considerable interests have focused on the development of high-efficiency electric energy storage devices and systems to meet the increasing demand for renewable energy. ${ }^{1-4}$ Among the electrical energy storage devices, dielectric capacitors have attracted much attention due to their applications in decoupling and DC-link capacitors, AC/DC converters, and portable power electronics for the advantages of high operation voltage, high power density, and low weight and volume. ${ }^{4-7}$ For a dielectric capacitor, high dielectric constant, high breakdown electric field, as well as low remnant polarization are desired for achieving high recoverable energy density $W_{r e c}$ and energy conversion efficiency $\eta .^{8,9}$ Although high-k dielectric oxide films exhibit high breakdown strength of 3-12 MV/cm and almost fully recoverable energy efficiency, ${ }^{6}$ the relatively low dielectric constant on the order of ten leads to a moderate recoverable energy density around $1-2 \mathrm{~J} / \mathrm{cm}^{3} .^{7}$ Ferroelectrics are promising for higher energy density than linear dielectrics, since their dielectric constant is larger than that of the linear dielectrics by one or two order in magnitude. ${ }^{7,10-12}$ Among them, the $\mathrm{PbZrO}_{3}$-based ferroelectric films, such as $(\mathrm{Pb}, \mathrm{La})(\mathrm{Zr}, \mathrm{Ti}) \mathrm{O}_{3}$, $(\mathrm{Pb}, \mathrm{La})(\mathrm{Zr}, \mathrm{Sn}, \mathrm{Ti}) \mathrm{O}_{3}$, and $(\mathrm{Pb}, \mathrm{Nb})(\mathrm{Zr}, \mathrm{Sn}, \mathrm{Ti}) \mathrm{O}_{3}$, exhibit high energy density $\left(10-85 \mathrm{~J} / \mathrm{cm}^{3}\right)$ at room temperature, and have been considered the most promising candidates for applications in high-power energy storage. ${ }^{13-22}$ However, the high energy density $\left(\sim 85 \mathrm{~J} / \mathrm{cm}^{3}\right)$ only can be achieved at room temperature due to lower breakdown voltage at higher temperature, ${ }^{22}$ and by far, the vast majority of investigations on $\mathrm{PbZrO}_{3}$-based ferroelectric thin films are focused on the

\footnotetext{
${ }^{\text {a) }}$ Authors to whom correspondence should be addressed. Electronic addresses: chunrui.ma@mail.xjtu.edu.cn and m.liu@mail.xjtu.edu.cn
}

energy storage properties at room temperature. In practical applications, the device operation temperature may fluctuate within a certain range. For example, the working temperature is up to $\sim 140^{\circ} \mathrm{C}$ for power electrics in hybrid electric vehicles. ${ }^{8,16}$ This makes thermal stability of the energy storage capacitors an important parameter and has prompted some interesting studies recently.

$\mathrm{Pb}_{0.96} \mathrm{La}_{0.04} \mathrm{Zr}_{0.98} \mathrm{Ti}_{0.02} \mathrm{O}_{3}$ antiferroelectric thin film exhibits a weakly temperature-dependent $W_{\text {rec }}$ of over $10 \mathrm{~J} / \mathrm{cm}^{3}$ from room temperature to $225^{\circ} \mathrm{C}$. Moreover, $\mathrm{PbZrO}_{3}$ and $\mathrm{Pb}_{0.97} \mathrm{La}_{0.02} \mathrm{Zr}_{0.95} \mathrm{Ti}_{0.05} \mathrm{O}_{3}$ antiferroelectric film also exhibit a good temperature stability of energy density. However, the $W_{\text {rec }}$ of the $\mathrm{Pb}$-based antiferroelectric thin film is usually less than $\sim 20 \mathrm{~J} / \mathrm{cm}^{3}$, because of the low breakdown strength in a wide temperature range. ${ }^{9,22,23}$ The need for increased $W_{\text {rec }}$ and $\eta$ in a wide temperature range has motivated the efforts of exploring relaxor ferroelectric materials, a subclass of ferroelectrics. The domain sizes of relaxor ferroelectrics are on order of nanometers instead of micrometers like those of typical ferroelectric materials, leading to smaller coercive field and lower remnant polarization in the typical $P-E$ loop. Relaxor ferroelectric system also has an important superiority in the Curie temperature becoming broad, and hence its properties are not highly dependent on temperature. Polycrystalline $\mathrm{Pb}_{0.92} \mathrm{La}_{0.08} \mathrm{Zr}_{0.52} \mathrm{Ti}_{0.48} \mathrm{O}_{3}$ (PLZT) relaxor ferroelectric film $(1-3 \mu \mathrm{m})$ exhibits a good thermal stability of energy density $\left(\sim 22 \mathrm{~J} / \mathrm{cm}^{3}\right)$ from room temperature to $200{ }^{\circ} \mathrm{C}^{8}{ }^{8}$ However, the polycrystalline film quality limits its breakdown strength, and hence induce a low $W_{\text {rec }}{ }^{9}$ In the polycrystalline film, there are many defects, grain boundaries, crystal orientations or fine pores, which not only assist in electron penetrating the film, but also influence its thermostability due to the defects that are 
very sensitive to temperature. For example, the grain boundary can change the control mechanism of electrical behavior with increasing temperature. ${ }^{24}$ Compared to the polycrystalline films, the epitaxial film, as an alternative for single crystal, can reduce the influence of defects and hence improve the thermostability of energy storage properties. It has been reported that the density of defects is reduced, and the mobility of the domain wall is improved in epitaxial PLZT thin films. ${ }^{25}$ This means that the higher energy density can be expected in epitaxial thin films, together with comparable or even better thermal stability. Motivated by this, we have investigated the energy density of the epitaxial PLZT thin films of $\sim 880 \mathrm{~nm}$ in thickness in the temperature range from room temperature to $180^{\circ} \mathrm{C}$ and found that a high energy density up to $\sim 31 \mathrm{~J} / \mathrm{cm}^{3}$ can be achieved in the entire range of the temperature, which represents a $41 \%$ enhancement relative to the polycrystalline case. $^{8}$

PLZT films of thickness $880 \mathrm{~nm}$ were fabricated using a pulsed laser deposition (PLD) system with $\mathrm{KrF}$ excimer laser (wavelength of $248 \mathrm{~nm}$ and pulse width of $25 \mathrm{~ns}$ ). The films were grown at $680{ }^{\circ} \mathrm{C}$ under 150 mTorr oxygen partial pressure on (001) $\mathrm{Nb}$ doped $\mathrm{SrTiO}_{3}$ (Nb:STO). The laser pulse energy density was around $2 \mathrm{~J} / \mathrm{cm}^{2}$ and the repetition rate was $5 \mathrm{~Hz}$. After the deposition, the films were in situ annealed under 350 Torr oxygen pressure at the growth temperature for $20 \mathrm{~min}$ to reduce oxygen vacancies before natural cooling down to room temperature. Platinum top electrodes with a thickness of $100 \mathrm{~nm}$ were deposited by sputtering through a shadow mask to define a set of square top electrodes of $200 \mu \mathrm{m}$ side length. The samples with Pt top electrodes were annealed at $450{ }^{\circ} \mathrm{C}$ in air for $5 \mathrm{~min}$ for electrode conditioning. The crystallinity of PLZT thin films was characterized using a highresolution X-ray diffraction system (HRXRD, PANalytical $X^{\prime}$ Pert MRD). The microstructure of the sample was characterized using a high resolution transmission electron microscopy (HRTEM, Jeol JEM-2100 TEM). A Radiant Technologies Precision Multiferroic tester with a heater station was used to determine the energy storage properties of PLZT thin films at a different temperature. The steady-state current density at different temperatures was characterized using this system.

Figure 1(a) shows a typical XRD $\theta-2 \theta$ profile of the PLZT thin film on the $(001) \mathrm{Nb}$ :STO substrate. Only $(00 l)$ can be found in the $\theta-2 \theta$ scan, revealing that the PLZT thin film is $c$-axis oriented. The rocking curve of the PLZT (001) peak was taken and the full width at half-maximum (FWHM) value (the inset of Figure $1(\mathrm{a})$ ) is around $0.38^{\circ}$, indicating that the PLZT thin film has a high quality. The $\varphi$ scan measurements were used to determine the in-plane epitaxial relationship between the PLZT thin film and the Nb:STO substrate. Figure 1(b) illustrates the $\{011\}$ reflections of the PLZT and Nb:STO, respectively. The fourfold symmetry reflections with well-defined sharp peaks are clearly seen, indicating that the PLZT films have a good single crystallinity and excellent epitaxial nature. The biaxial epitaxial relationship with the substrate is $[100]_{\mathrm{PLZT}} / /[100]_{\mathrm{Nb}: \mathrm{STO}}$ (in-plane) and $(001)_{\mathrm{PLZT}} / /(001)_{\mathrm{Nb}: \mathrm{STO}}$ (out-of-plane). Based on the reciprocal space mappings (RSMs) data (Figures 1(c) and 1(d)), the in-plane and out-ofplane lattice constant of PLZT film is estimated to be $4.060 \AA$ and $4.065 \AA$, respectively, which is comparable to the lattice constant of the bulk PLZT material $c=4.068 \pm 0.005 \AA{ }^{26}$ This indicates that the interface strain induced by the lattice mismatch between the film and the substrate is almost relaxed at the film thickness of $\sim 880 \mathrm{~nm}$ through the formation of misfit dislocations and other defects. Figure 1(e) displays an overview microstructure of the PLZT films on the Nb:STO substrates, viewed along the [010] zone axis of Nb:STO. Au and $\mathrm{Pt}$ are deposited on the film to prevent the accumulation of charge at the film during the TEM study. The sharp filmsubstrate interface is clearly visible. The columnar structured growth dominates in the PLZT thin films as reported previously, ${ }^{8,25}$ and the columnar grains in the PLZT films are mostly perpendicular to the substrate. In the columnar structured ferroelectric thin films, the uniformly polarized crystallites could be easily realized. ${ }^{27,28}$ The selected-area electron diffraction
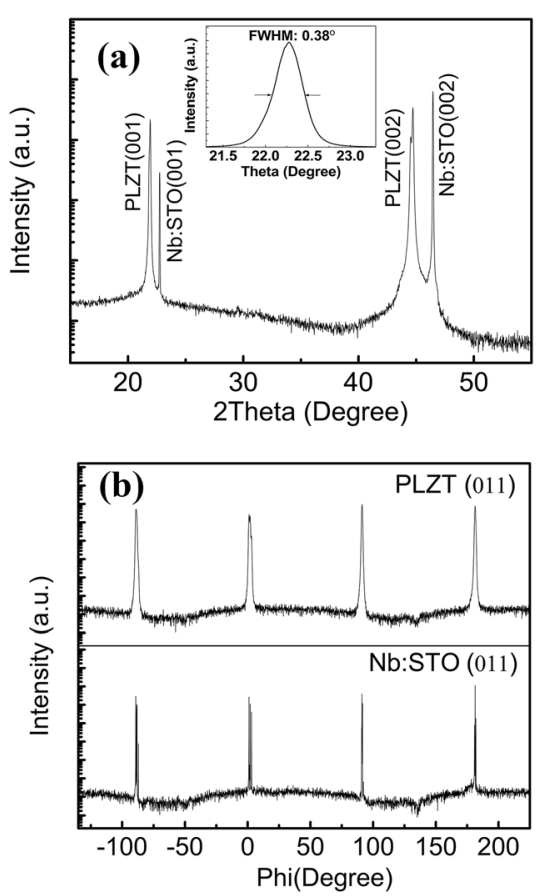
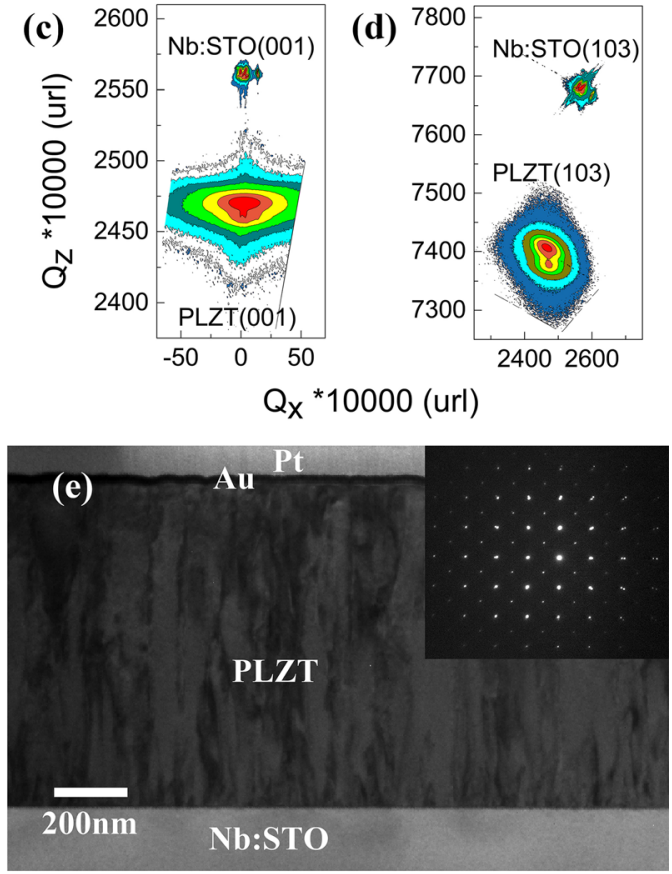

FIG. 1. (a) Typical XRD pattern of PLZT films grown on (001) Nb:STO substrate. The inset of (a) is a rocking curve from the (001) reflection for the PLZT Film. (b) The $\varphi$ scans taken around the $\{011\}$ diffraction of PLZT film and Nb:STO substrate. (c) and (d) The reciprocal space mappings taken around from the symmetric (001) and asymmetric (103) reflections of PLZT film and Nb:STO substrate, respectively. (e) The cross section TEM images and the SAED patterns taken from the areas covering the interfaces between the films and substrates. 
(SAED) pattern of a PLZT/Nb:STO sample was recorded along the [010] zone axis of Nb:STO. The splitting of diffraction spots of PLZT and Nb:STO can be detected, indicating that the strain relaxation occurs in the PLZT/Nb:STO heterostructure, which is consistent with the XRD measurement.

Figure 2(a) shows the $P-E$ loops of epitaxial PLZT thin film measured at room temperature $\left(\sim 25^{\circ} \mathrm{C}\right)$ with applied voltages of $120 \mathrm{~V}, 160 \mathrm{~V}$, and $200 \mathrm{~V}$, respectively. The data reveals the epitaxial PLZT thin film has well-defined and slim hysteresis curves, which is characteristic of relaxor ferroelectric materials and favorable to low-loss energy storage. As shown in Figures 2(b) and 2(c), both the maximum polarization $P_{\max }$ and the remnant polarization $P_{r}$ increase with increasing applied electric field because a larger applied electric field leads to an increased domain wall movement and better electric dipole alignment in the epitaxial PLZT thin films. Both $W_{\text {rec }}$ (as highlighted in Figure 2(a) by the shaded area) and $\eta$ are plotted in Figure 2(d) as a function of the applied electric field. It is found that the $W_{\text {rec }}$ steadily increases, and the $\eta$ almost keeps a constant with the increase of applied electric field. The highest values of $W_{\text {rec }} \sim 31.5$ $\mathrm{J} / \mathrm{cm}^{3}$ and $\eta \sim 76.4 \%$ were demonstrated, respectively, at the upper limit of the applied electric field $E_{\max }=2.27 \mathrm{MV} / \mathrm{cm}$ in this work. It is found that the breakdown strength is higher than that in the polycrystalline films with a comparable thickness $(\sim 1 \mu \mathrm{m}, 2.0 \mathrm{MV} / \mathrm{cm}),{ }^{8}$ thus making the $W_{\text {rec }}$ and $\eta$ remarkably improved by $46.5 \%$ and $21.2 \%$, respectively. The enhanced breakdown strength probably relates to the improved film quality. From the microstructure analysis (Figure 1), there are no grain boundaries, porosity in the epitaxial film, which prevents the electric tree from penetrating the film by grain boundaries or porosity and thus increases the breakdown strength. ${ }^{9,29}$

To investigate the thermal stability of epitaxial PLZT thin films, the temperature-dependent $P-E$ loops at the fixed $E_{\max }=2.27 \mathrm{MV} / \mathrm{cm}$ are measured and shown in Figure 3(a). Both the maximum polarization $P_{\max }$ (black) and the remnant polarization $P_{r}$ (red) increase as the operating temperature increase (Figure 3(b)). This trend can be explained by the thermal activation energy theory of the domain wall movement. The higher thermal energy at an increased temperature facilitates the ferroelectric domain movement and hence increases the maximum and remnant polarizations. ${ }^{30,31}$ The calculated $W_{\text {rec }}$ and $\eta$ at different temperatures in the tested temperature range are summarized in Figure 3 (c). The former (black) remains approximately a constant at around $31 \mathrm{~J} / \mathrm{cm}^{3}$ in almost the whole temperature range except a slight decrease by $6.3 \%$ at $180^{\circ} \mathrm{C}$. In contrast, the latter (blue) decreases monotonically from $76.4 \%$ to $53.2 \%$ as the temperature increased from room temperature to $180^{\circ} \mathrm{C}$ due to the increasing contribution of the electric conduction part. ${ }^{32}$ This thermal stability of the energy density is remarkable and represents a significant improvement over that in previous reports on ferroelectric film capacitors. For example, a decreasing trend in the energy density from $22 \mathrm{~J} / \mathrm{cm}^{3}$ to $18 \mathrm{~J} / \mathrm{cm}^{3}$ as temperature increased from $20^{\circ} \mathrm{C}$ to $120^{\circ} \mathrm{C}$ was reported by Wang et al. on polycrystalline PLZT (2/98/2) thick films $(2 \mu \mathrm{m})$ due to the decrease of the maximum polarization and the increase of the coercive field. ${ }^{33}$ Also, a decreasing energy density from $53 \mathrm{~J} / \mathrm{cm}^{3}$ to $37 \mathrm{~J} / \mathrm{cm}^{3}$ as the temperature increases from $25^{\circ} \mathrm{C}$ to $150^{\circ} \mathrm{C}$ was reported by Ma et al. on polycrystalline PLZT (8/95/5) antiferroelectric thick film $(1 \mu \mathrm{m})$ due to the electric fieldinduced antiferroelectric to ferroelectric transition that is very sensitive to temperature. Although a stable temperature independent energy storage densities were achieved in polycrystalline PLZT (8/52/48) relaxor ferroelectric films, the relatively poor film quality, like grain boundaries, crystalline orientation and cracks, induce lower breakdown strength in wide temperature range, and hence lower energy density $\left(21.5 \mathrm{~J} / \mathrm{cm}^{3}, 1 \mu \mathrm{m}\right){ }^{8}$ Compared to the polycrystalline film, the high quality epitaxial PLZT film effectively reduces the effect from these exterior factors, which can be easily affected by temperature, and hence makes the breakdown
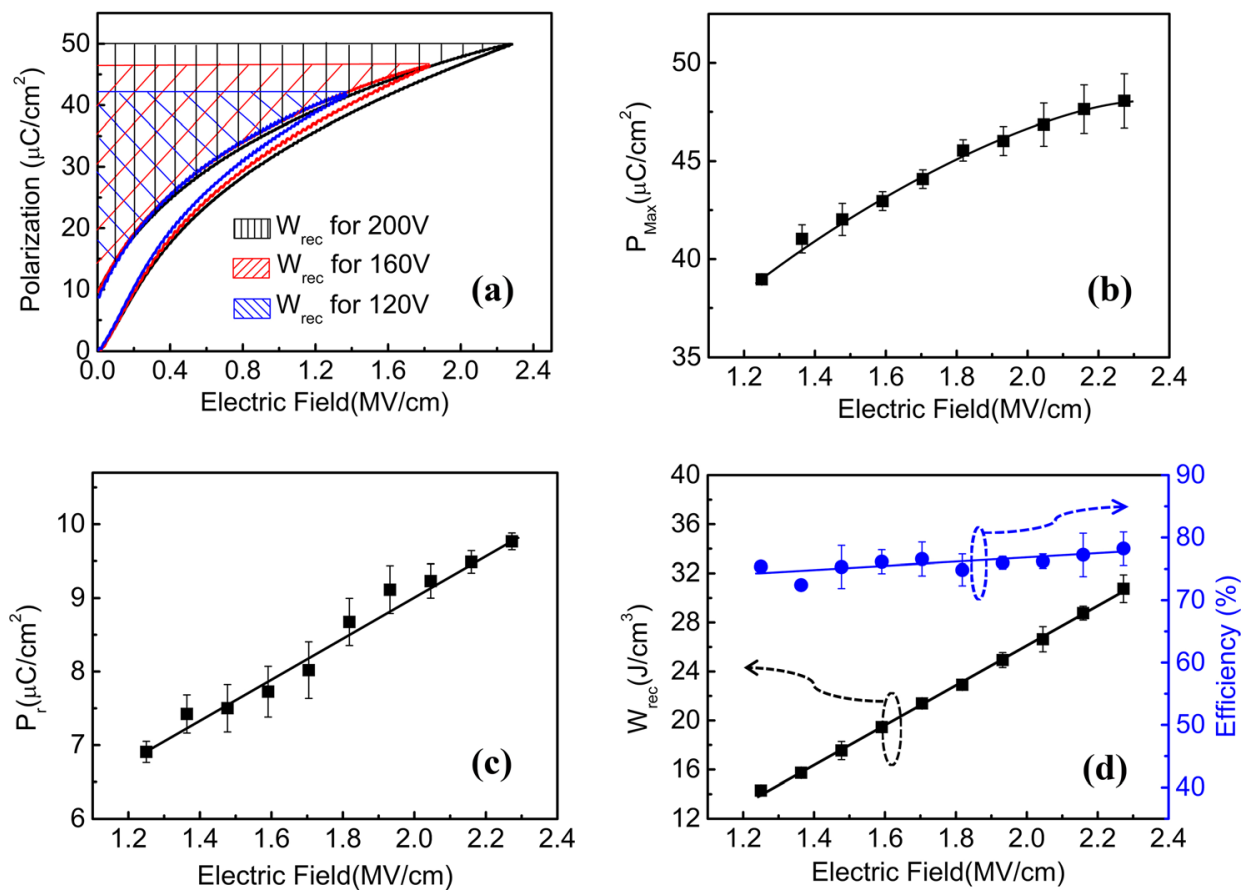

FIG. 2. (a) $P-E$ loops at different applied electric field. (b) and (c) Electric field dependent maximum polarization $P_{\max }$ and remnant polarization $P_{r}$, respectively. (d) Electric field dependent recoverable energy density (black) and efficiency (blue) of epitaxial PLZT thin films. 

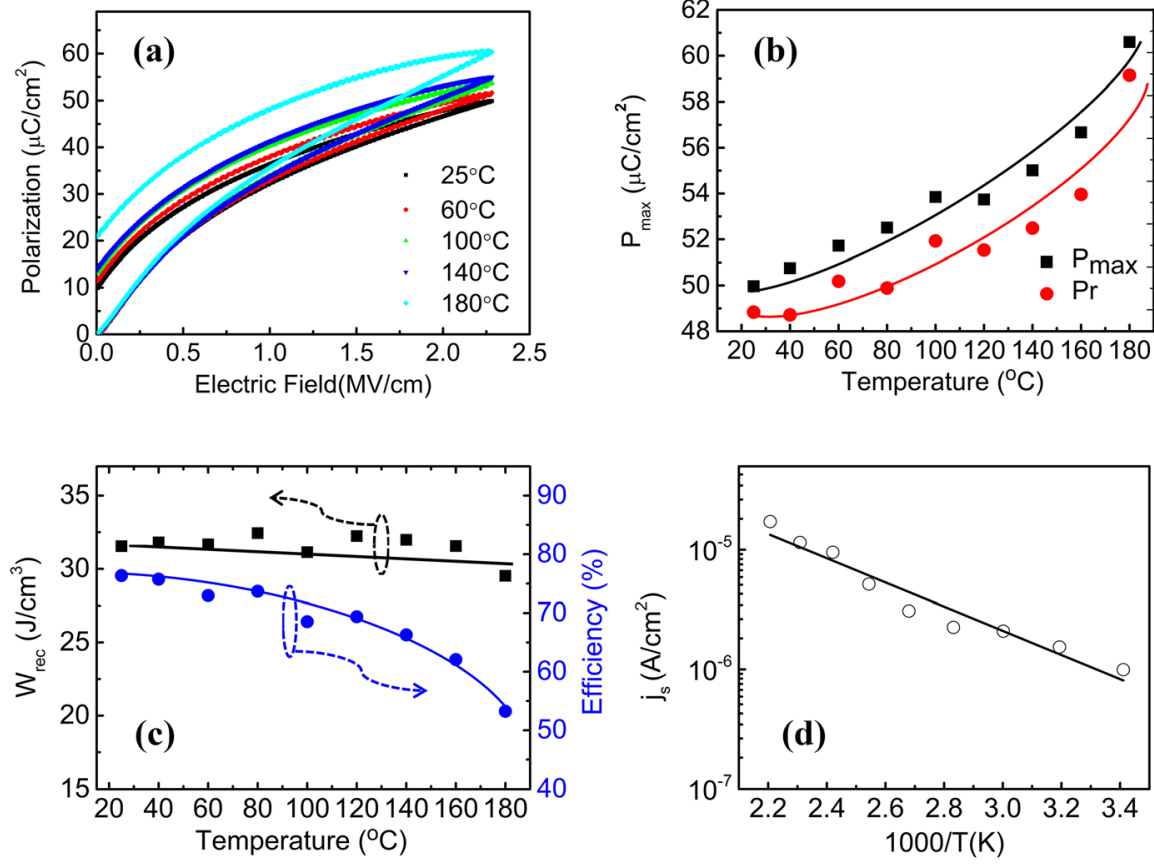

FIG. 3. (a) $P-E$ loops at a different temperature with a fixed electric field $2.27 \mathrm{MV} / \mathrm{cm}$. (b) Temperature dependent maximum polarization $P_{\max }$ (black) and remnant polarization $P_{r}$ (red). (c) Temperature dependent recoverable energy density (black) and efficiency (blue) of epitaxial PLZT thin films. (d) Steady-state leakage current density as a function of temperature. strength increase in wide temperature range, leading to a superior thermostability of energy density. In order to further understand the observed thermal stability of the energy density, the steady-state current density $j_{s}$ of the epitaxial PLZT thin film at different temperatures was extracted from the leakage current density $\left(j=j_{s}+j_{0} t^{-n}\right)$ measured $1 \mathrm{~s}$ after the application of the electric field $\left(E_{d c}=0.11 \mathrm{MV} / \mathrm{cm}\right)$. As shown in Figure 3(d), the leakage current density increases as the operating temperature is increasing, since the thermal activation energy assists the electron hopping in the films, and hence increase the leakage current density. It is noted that the leakage current density in epitaxial PLZT film is a little larger than that in polycrystalline PLZT film due to the reduced activation energy $\sim 0.20 \mathrm{eV}(\sim 0.29 \mathrm{eV}$ for the polycrystalline PLZT films), obtained by fitting the data using Arrhenius equation $j_{s}=C e^{-\frac{E_{a}}{k T}}$, where $C$ is a constant, $E_{a}$ is the activation energy, $k$ is the Boltzmann constant. ${ }^{34}$ The decreased activation energy results from the high mobility of dipole domain in the epitaxial PLZT film, which can assist electron transport. Although the reduced activation energy makes the leakage current density increase, it is low enough to support the use of our thin-film capacitors over a wide range of temperature.

The dynamic hysteresis and energy storage properties of the epitaxial PLZT thin film over frequency from $100 \mathrm{~Hz}$ to $1 \mathrm{MHz}$ at room temperature under $1.14 \mathrm{MV} / \mathrm{cm}$ electric field are shown in Figure 4. From Figure 4(a), it can be seen that the $P$ - $E$ loops become thinner with the increase of operating frequency due to the interfacial space charge layers widening the $P-E$ loop at low frequency and induce larger hysteresis loss, leading to the lower $\eta .^{21}$ The $P_{\max }$ and $P_{r}$ of the epitaxial PLZT thin film decrease as the frequency increase due to the contribution of space charge polarization decrease, which is the same as the behavior of PLZT (4/98/2) thin film. ${ }^{21}$ The $\eta$ almost keeps a constant $(\sim 75 \%)$ when the frequency is above $1 \mathrm{kHz}$, at which there is an absence of space charge polarization. This behavior is similar to the PLZT (4/98/2) and PLZT $(2 / 95 / 5)$, expect the relative higher $\eta$ due to reduced defects through epitaxy. ${ }^{9,21}$ In contrast, the $W_{\text {rec }}$ fluctuates between $11.6 \mathrm{~J} / \mathrm{cm}^{3}$ and $10.6 \mathrm{~J} / \mathrm{cm}^{3}$, which demonstrates that the $W_{\text {rec }}$ is weakly frequency-dependent. The data measured in the test temperature range $\left(25^{\circ} \mathrm{C}-180^{\circ} \mathrm{C}\right)$ exhibit similar behaviors. These results indicate that the epitaxial PLZT thin film is a better promising candidate for the capacitive energy storage in a wide temperature and frequency range.

In summary, high quality epitaxial PLZT thin film capacitors were obtained on conductive $\mathrm{Nb}$ :STO substrates (bottom electrode) using PLD, followed with Pt top electrode deposition using sputtering. Thermal stability of the energy
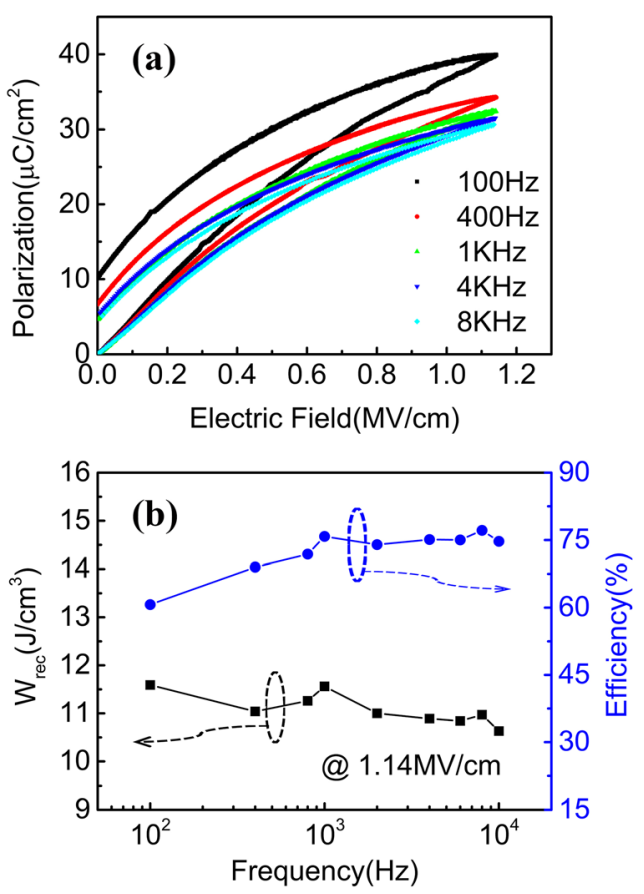

FIG. 4. (a) Selected $P-E$ loops at different frequencies with a fixed electric field $1.14 \mathrm{MV} / \mathrm{cm}$. (b) Frequency-dependent energy storage density (black) and efficiency (blue) of the epitaxial PLZT thin film. 
storage properties was evaluated in the temperature range from room temperature to $180^{\circ} \mathrm{C}$, relevant to operation temperatures of the power systems in the hybrid electric vehicles $\left(\sim 140^{\circ} \mathrm{C}\right)$ and other devices. High recoverable energy density up to $31 \mathrm{~J} / \mathrm{cm}^{3}$ was observed for the epitaxial PLZT thin films, which represents a remarkable improvement of $41 \%$ over that of the polycrystalline counterpart. Moreover, the recoverable energy density was found almost temperature independent in the tested temperature range due to the higher mobility of dipole domains at a much reduced defect concentration through epitaxy. These results indicate that the epitaxial PLZT thin films are a promising candidate for energy storage in a wide operation temperature and frequency range.

This research was supported by the China Postdoctoral Science Foundation (No. 2015M582649), National "973" projects of China (No. 2015CB654903), Natural Science Foundation of China (Nos. 51202185 and 51390472), Shaaxi Province Postdoctoral Science Foundation, and the Fundamental Research Funds for the Central Universities. J.W. acknowledges the support in part by NASA Contract NNX13AD42A and NSF Contract NSF-DMR1509484.

${ }^{1}$ J. Xu, D. H. Lee, and Y. S. Meng, Abs. Pap. Am. Chem. Soc. 244, 596 (2012).

${ }^{2}$ X. W. Cui, J. Chen, T. F. Wang, and W. X. Chen, Sci. Rep. 4, 5310 (2014).

${ }^{3}$ D. Larcher and J. M. Tarascon. Nat. Chem. 7, 19-29 (2015).

${ }^{4}$ B. J. Chu, X. Zhou, K. L. Ren, B. Neese, M. R. Lin, Q. Wang, F. Bauer, and Q. M. Zhang, Science 313, 334-336 (2006).

${ }^{5}$ S. A. Sherrill, P. Banerjee, G. W. Rubloff, and S. B. Lee, Phys. Chem. Chem. Phys. 13, 20714-20723 (2011).

${ }^{6}$ J. W. McPherson, K. Jinyoung, A. Shanware, H. Mogul, and J. Rodriguez, IEEE Trans. Electron Devices 50, 1771-1778 (2003).

${ }^{7}$ N. Ortega, A. Kumar, J. F. Scott, D. B. Chrisey, M. Tomazawa, S. Kumari, D. G. Diestra, and R. S. Katiyar, J. Phys.: Condens. Matter 24, 445901 (2012).

${ }^{8}$ S. Tong, B. Ma, M. Narayanan, S. Liu, R. Koritala, U. Balachandran, and D. Shi, ACS Appl. Mater. Interfaces 5, 1474-1480 (2013).
${ }^{9}$ C. Liu, S. X. Lin, M. H. Qin, X. B. Lu, X. S. Gao, M. Zeng, Q. L. Li, and J. M. Liu, Appl. Phys. Lett. 108, 112903 (2016).

${ }^{10}$ L. Zhu and Q. Wang, Macromolecules 45, 2937-2954 (2012).

${ }^{11}$ H. Ogihara, C. A. Randall, and S. Trolier-McKinstry, J. Am. Ceram. Soc. 92, 1719-1724 (2009).

${ }^{12}$ X. Hao, J. Adv. Dielectr. 3, 1330001 (2013).

${ }^{13}$ J. Zhai, M. H. Cheung, Z. K. Xu, X. Li, H. Chen, E. V. Colla, and T. B. Wu, Appl. Phys. Lett. 81, 3621 (2002).

${ }^{14}$ J. Parui and S. B. Krupanidhi, Appl. Phys. Lett. 92, 192901 (2008).

${ }^{15}$ M. S. Mirshekarloo, K. Yao, and T. Sritharan, Appl. Phys. Lett. 97, 142902 (2010).

${ }^{16}$ B. H. Ma, D. K. Kwon, M. Narayanan, and U. Balachandran, J. Mater. Res. 24, 2993-2996 (2009).

${ }^{17}$ Y. Wang, X. Hao, J. Yang, J. Xu, and D. Zhao, J. Appl. Phys. 112, 034105 (2012).

${ }^{18}$ J. Ge, X. Dong, Y. Chen, F. Cao, and G. Wang, Appl. Phys. Lett. 102, 142905 (2013).

${ }^{19}$ S. E. Young, J. Y. Zhang, W. Hong, and X. Tan, J. Appl. Phys. 113, 054101 (2013).

${ }^{20}$ B. H. Ma, Z. Q. Hu, R. E. Koritala, T. H. Lee, S. E. Dorris, and U. Balachandran, J. Mater. Sci.:Mater. Electron. 26, 9279-9287 (2015).

${ }^{21}$ H. Pan, Y. Zeng, Y. Shen, Y. H. Lin, and C. W. Nan, J. Appl. Phys. 119, 124106 (2016).

${ }^{22}$ Z. Q. Hu, B. H. Ma, R. E. Koritala, and U. Balachandran, Appl. Phys. Lett. 104, 263902 (2014).

${ }^{23}$ J. Ge, D. Remiens, X. L. Dong, Y. Chen, J. Costecalde, F. Gao, F. Cao, and G. S. Wang, Appl. Phys. Lett. 105, 112908 (2014).

${ }^{24}$ Z. H. Zhou, J. M. Xue, W. Z. Li, J. Wang, H. Zhu, and J. M. Miao, Appl. Phys. Lett. 85, 804-806 (2004).

${ }^{25}$ C. R. Ma, B. H. Ma, S. B. Mi, M. Liu, and J. Wu, Appl. Phys. Lett. 104, 162902 (2014).

${ }^{26}$ R. N. P. Choudhary and J. Mal, Mater. Lett. 54, 175-180 (2002).

${ }^{27}$ S. Sriram, M. Bhaskaran, D. R. G. Mitchell, K. T. Short, A. S. Holland, and A. Mitchell, Microsc. Microanal. 15, 30-35 (2009).

${ }^{28}$ A. Gruverman, O. Auciello, and H. Tokumoto, Annu. Rev. Mater. Sci. 28, 101-123 (1998).

${ }^{29}$ K. Yao, S. T. Chen, M. Rahimabady, M. S. Mirshekarloo, S. H. Yu, F. E. H. Tay, T. Sritharan, and L. Lu, IEEE Trans. Ultrason. Ferroelectr. 58, 1968-1974 (2011).

${ }^{30}$ W. J. Merz, Phys. Rev. 95, 690-698 (1954).

${ }^{31}$ R. C. Miller and G. Weinreich, Phys. Rev. 117, 1460-1466 (1960).

${ }^{32}$ E. Brown, C. Ma, J. Acharya, B. Ma, J. Wu, and J. Li, ACS Appl. Mater. Interfaces 6, 22417-22422 (2014).

${ }^{33}$ Y. Wang, X. H. Hao, and J. B. Xu, J. Mater. Res. 27, 1770-1775 (2012).

${ }^{34}$ S. M. Cho and D. Y. Jeon, Thin Solid Films 338, 149-154 (1999). 